\title{
Aquisição da habilidade motora rebater na Educação Física escolar: um estudo das dicas de aprendizagem como conteúdo de ensino
}

CDD. 20.ed. 796.017

\author{
Sérgio Roberto SILVEIRA* \\ Luciano BASSO ${ }^{* *}$ \\ Andrea Michele FREUDENHEIM ${ }^{* *}$ \\ Umberto Cesar CORRÊA** \\ Marilda Gonçalves FERREIRA*** \\ Go TANI ${ }^{* *}$ \\ *Coordenadoria de \\ Gestão da Educação \\ Básica, Secretaria de \\ Estado da Educação \\ de São Paulo. \\ **Escola de Educaçãa \\ Física e Esporte, \\ Universidade de São \\ Paulo. \\ ${ }^{* * *}$ Rede Pública \\ Estadual de Ensino de \\ São Paulo.
}

\section{Resumo}

0 objetivo do estudo foi investigar o efeito das dicas verbais na aquisição da habilidade rebater na Educação Física Escolar (EFE), com foco de atenção no seu aspecto perceptivo e motor. Participaram do estudo 84 alunos de três turmas de uma escola (faixa etária entre seis a oito anos). Cada turma correspondeu a um grupo: sem dicas, com dica perceptiva e com dica motora. 0 experimento teve três fases: pré-teste, aulas de EFE e pós-teste. 0 grupo com dica perceptiva apresentou melhores resultados. Portanto, verificou-se o efeito das dicas relacionado com a especificidade da tarefa no que se refere às suas demandas de processamento. Assim concluiu-se que a dica de aprendizagem relacionada à especificidade da tarefa mostrou o seu potencial como um conhecimento que orienta os alunos em direção à melhoria da qualidade do movimento, confirmando a possibilidade de sua inclusão como conteúdo de ensino da EFE.

PalavRAs-Chave: Educação física escolar; Aprendizagem do movimento; Dicas verbais; Conteúdo de ensino.

\section{Introdução}

A compreensão de que a educação física escolar (EFE) tem por finalidade disseminar conhecimentos historicamente produzidos e sistematizados relativos à cultura de movimento, ou seja, o jogo, o esporte, a dança, a ginástica e o exercício, tem estado presente em muitas abordagens propostas ao longo das últimas décadas no nosso país. Esses conhecimentos correspondem a um patrimônio cultural que deve ser socializado nas aulas de EFE, com vistas a atender as finalidades da educação escolarizada ${ }^{1}$. No entanto, surge o questionamento de como organizar o ensino acerca desse patrimônio de modo a garantir uma aprendizagem significativa para o aluno. TANI ${ }^{1-3}$ propóe que esses conhecimentos poderiam ser organizados em três aprendizagens relativas à cultura de movimento: aprendizagem do movimento, através do movimento e sobre o movimento.
O presente estudo centra-se na aprendizagem do movimento, cujo desenvolvimento no contexto da educação escolarizada é de inteira responsabilidade da EFE. De acordo com HALVERSON ${ }^{4}$, aprender a mover-se envolve contínuo desenvolvimento da capacidade de usar o corpo efetivamente e harmoniosamente com crescente evidência de controle e qualidade no movimento. Envolve também o desenvolvimento da capacidade de mover-se numa variedade de maneiras, em situaçōes esperadas e inesperadas e em tarefas crescentemente complexas.

A aprendizagem do movimento é, portanto, um processo complexo que requer longo tempo e quantidade de prática 5 . Mas é exatamente por esse motivo que a possibilidade de sua efetiva implementação no contexto da educação escolarizada é colocada "em xeque": como promover a melhoria 
da qualidade do movimento durante as aulas de educação física quando se considera a realidade do sistema de ensino quanto a dias e horas de aulas semanais, número de alunos por classe, espaços e materiais didáticos disponíveis?

Uma possível solução para esse problema foi proposta por $\mathrm{TANI}^{1,3}$ ao apresentar outra dimensão dessa mesma aprendizagem: o conhecimento sobre como melhorar a qualidade do movimento. Conhecer acerca de como melhorar a qualidade do movimento significa ser capaz de identificar os processos e procedimentos essenciais à aquisição de habilidades motoras. Dessa forma, a aquisição desse conhecimento na escola possibilitaria ao aluno a sua utilização em todas as oportunidades de prática fora da escola, para melhorar a habilidade ${ }^{1,3}$. Assumese, portanto, que ensinar os conhecimentos sobre como melhorar a qualidade do movimento pode se constituir um dos conteúdos fundamentais da EFE.

O processo de melhoria da qualidade do movimento ou a aquisição de habilidades motoras é o objeto de estudo do campo de investigação denominado Aprendizagem Motora, que tem um longo histórico de pesquisas realizadas. Assim, buscou-se na Aprendizagem Motora um saber relativo à melhoria da qualidade do movimento que pudesse ser ensinado na EFE. Essa busca resultou nas dicas de aprendizagem, com vasto número de estudos realizados, sobretudo, como estratégias cognitivas para a canalização de atenção na aquisição de habilidades motoras ${ }^{6-11}$.

Dicas verbais podem ser entendidas como uma forma de instrução verbal curta e direcionada, com alguns pontos-chave, para orientar o aprendiz aos elementos importantes da habilidade motora a ser executada ${ }^{6,12}$. As dicas têm por função guiar e estimular a imaginação do aprendiz para a ação, ressaltando os componentes-chaves da habilidade motora, ou seja, configuram-se como um meio alternativo de comunicar a informação significativa da tarefa a ser executada $a^{6,10,12-14}$.

Apesar do histórico interesse de estudo relacionado às dicas verbais na Aprendizagem Motora, pesquisas que investiguem sua efetividade no contexto da EFE como conteúdo de ensino são ainda escassas. Verificam-se apenas dois estudos realizados com crianças no contexto escolar: um deles utilizou dois exercícios de solo de ginástica: parada de mãos e rolamento para frente ${ }^{13}$; o outro, a rebatida ${ }^{15}$. Em ambos os estudos, os resultados mostraram que o fornecimento das dicas conduziu a uma significativa melhora no desempenho.

O presente estudo se desenvolveu numa escola da rede pública estadual de ensino de São Paulo, com crianças nas séries iniciais do ensino fundamental. Dessa forma, o conteúdo de ensino relacionou-se aos movimentos fundamentais próprios dessa fase de desenvolvimento ${ }^{16}$. A escolha recaiu na habilidade motora rebater que, apesar de atender ao requisito anterior, em razão de fatores culturais, não é muito praticada na nossa sociedade, comparativamente, a outras habilidades como o chutar e o arremessar. Sob o ponto de vista do estudo, isso se constitui um importante fator, pois deixa uma ampla possibilidade para se verificar a ocorrência efetiva da aprendizagem.

A habilidade rebater refere-se à ação de propulsão de um objeto, utilizando diretamente o próprio corpo ou um implemento. Quando a propulsão se dá em relação a objeto em movimento, caso do presente estudo, o desafio para o aprendiz é perceber a trajetória e o "timing" de deslocamento do objeto, de modo a executar a ação motora no tempo correto ${ }^{17-18}$. Gallahue et al. ${ }^{19}$ destacam dois fatores que influenciam o rebater: a focalização e rastreamento da trajetória do objeto (uma bola, por exemplo) e a velocidade máxima do movimento no instante da rebatida.

Dentre as características que tornaram o movimento escolhido - rebater - relevante para o desenvolvimento do estudo junto às séries iniciais do ensino fundamental, que compreendeu alunos com idade entre seis e oito anos, destacam-se os seguintes aspectos: a) configura-se como habilidade manipulativa que representa ação motora com profundos significados no processo evolutivo do ser humano; b) configura-se como habilidade em grau de maior complexidade em relação ao rebater bola estacionária; c) é habilidade requerida em graus crescentes de complexidade, nas diferentes manifestações da cultura de movimento; d) é habilidade que requer informações que permitem ao aluno vislumbrar o como aprendê-las; e) nessa habilidade, deparase com dificuldades de execução que passam por demandas perceptivas e motoras; f) na habilidade, nota-se a presença de característica peculiar para o início da execução, possibilitando estabelecer hipóteses distintas na demanda de atenção seletiva para a respectiva tarefa: a bola partirá externamente ao aluno, levando-o, possivelmente, a necessitar de dicas de aprendizagem para canalizar sua atenção inicial no aspecto perceptivo, com vistas a acompanhar a trajetória da bola e realizar a ação ${ }^{20}$.

Em suma, diante do potencial que as dicas têm em favorecer a aquisição de habilidades motoras e da carência de estudos sobre o tema em ambientes escolares, o presente trabalho teve como objetivo 
investigar a utilização das dicas de aprendizagem como conteúdo de ensino para promover a aprendizagem do movimento. $\mathrm{Na}$ realidade, a dica pode ser vista, no presente trabalho, como um elemento de "dupla face”, ou seja, estratégia e conteúdo ao mesmo tempo. Conforme descrito anteriormente, tradicionalmente ela tem sido vista como uma estratégia de direcionar a atenção do aluno para promover a aprendizagem. Contudo, a informação nela contida a transforma também num conhecimento que tem o potencial de facilitar a melhora da qualidade do movimento. Com isso, o objetivo do presente estudo foi investigar se a apresentação da dica influencia a aprendizagem da habilidade de rebater. Ademais, considerando-se que

\section{Método}

\section{Tarefa de aprendizagem}

A tarefa motora consistiu em realizar rebatidas, o mais longe possível, de uma bola em movimento, lançada pelo professor de educação física, na linha média do corpo, na altura da cintura pélvica do aluno, à frente da base do rebatedor a uma distância de três metros.

\section{Materiais}

Utilizou-se uma bola de borracha (número 8) e um bastão de madeira com comprimento de 50 centímetros, com diâmetro de $20 \mathrm{~mm}$.

\section{Amostra}

Participaram do estudo 84 escolares, com idades entre seis anos (completos) e oito anos (até a véspera de completar nove), de três turmas de EFE de uma escola da rede pública estadual de ensino, ciclo I, Ensino Fundamental, em classes de tempo integral. Dessa maneira, os grupos de pesquisa compreenderam duas turmas de segundo ano e uma turma de terceiro ano. A participação dos alunos no estudo foi condicionada à assinatura de termo de consentimento por parte dos responsáveis. O projeto de pesquisa, com protocolo 2009/46, foi devidamente aprovado pelo Comitê de Ética da Escola de Educação Física e Esporte da Universidade de São Paulo. a identificação dos componentes-chaves da habilidade é fundamental para a eficácia das dicas, pretendeuse também investigar se o tipo de dica - perceptiva ou motora - pode influenciar diferentemente a aprendizagem do rebater.

Vale ressaltar que o presente estudo se caracterizou como uma pesquisa experimental de campo em que as características do contexto foram respeitadas na formação dos grupos, ou seja, cada grupo correspondeu aos alunos de uma turma de EFE. Portanto, esperou-se por uma heterogeneidade no nível de habilidade dos mesmos. Em razão disso, também se analisou a influência dos níveis de habilidade iniciais na aquisição da habilidade do rebater.

\section{Delineamento e procedimentos}

Mantendo-se o sigilo da identificação pessoal dos alunos, o delineamento seguiu o seguinte formato: G1 - grupo rebater sem dicas com 26 alunos; G2 - grupo rebater com dica perceptiva com 29 alunos; G3 - grupo rebater com dica motora com 29 alunos. As dicas perceptiva e motora foram, respectivamente, "olho na bola" e "rebater com o máximo de velocidade".

Os dados foram coletados no ambiente de uma quadra poliesportiva em duas fases: pré-teste e pósteste. As dicas foram aplicadas nas cinco aulas de EFE desenvolvidas entre as duas fases de coleta. As filmagens do pré-teste e do pós-teste foram efetuadas por uma câmera marca Sony CyberShot, modelo DSC-V3, posicionada a três metros lateralmente ao aluno. O programa Virtual Dub (versão 1.6), com 60 quadros por segundo, foi utilizado como "software" para o registro dos dados. O pós-teste foi realizado após a conclusão de uma semana e meia de aulas. No período compreendido entre o pré-teste e o pós-teste, as mesmas aulas de EFE foram ministradas para as três turmas com a utilização ou não de dicas, conforme o delineamento descrito acima, com base no planejamento realizado em conjunto entre o experimentador e o professor especialista responsável pela disciplina.

O desempenho foi analisado de acordo com: 1) estágio de desenvolvimento da habilidade de rebater; 2) tipo de rebatida; e 3) distância da rebatida. No 
tocante a primeira variável dependente, seguiu-se como critério para a classificação nos estágios o maior número de comportamentos observados registrados em uma ficha de checagem utilizada. Foram adotados os seguintes comportamentos: a) Estágio Inicial (I) - dificuldade em coincidir o movimento do bastão com a bola lançada; manutenção dos pés paralelos; falta ou pequeno giro do tronco; movimentação sem velocidade do braço; b) Estágio Elementar (E) - ação coincidente entre o rastreamento da bola e o golpe com o bastão, mas com pequena propulsão do objeto; execução da habilidade com pequeno afastamento anteroposterior das pernas; giro do tronco insuficiente e ação do braço incompleta e com pouca velocidade; c) Estágio Maduro (M) - atinge o bastão na bola com precisão; afastamento anteroposterior das pernas; giro do tronco e ação do braço suficiente para produzir uma movimentação com velocidade na rebatida. O estágio de desenvolvimento foi definido de acordo com o valor da moda de três tentativas.

Referente ao desempenho no que diz respeito ao tipo de rebatida considerou-se quatro estágios: 1 ) não acerto da bola; 2) acerto da bola com rebatida para trás; 3) acerto da bola com desvio à lateral e a frente; 4) acerto da bola para frente. O tipo de rebatida das três tentativas foi designado também de acordo com o valor da moda.

Finalmente, o desempenho relativo à rebatida foi avaliado de acordo com a distância que a bola alcançou, medido em metros. Ela foi definida com base na mediana das três tentativas.

\section{Resultados}

A distribuição dos alunos entre os estágios de desenvolvimento do rebater é apresentada na TABELA 1. É possível observar que no pré-teste todos os grupos tiveram a maioria dos alunos concentrada no estágio inicial de desenvolvimento da habilidade. Esses resultados são semelhantes no pós-teste, com exceção do grupo de dica perceptiva, em que os alunos mudam para os estágios elementar e maduro. Esse comportamento foi confirmado pelas análises inferenciais, uma vez que os testes de Kruskall Wallis

\section{Tratamento dos dados}

Para caracterizar o comportamento dos grupos foram utilizados valores modais para o estágio de desenvolvimento da habilidade e tipo de rebatida e valores medianos para as distâncias alcançadas nas rebatidas contempladas nos Resultados e Discussão.

Conduziu-se uma análise exploratória e descritiva dos dados. A distância da rebatida apresentou distribuição não normal para todos os grupos. Com isso, as análises inferenciais entre os valores do pré e pós-teste para as medidas de distância rebatida e padrão de movimento foram conduzidas por uma série de testes não paramétricos de Wilcoxon. As análises intergrupos foram conduzidas por uma série de testes não paramétricos de Kruskall Wallis, seguido do procedimento de comparações múltiplas apresentado por SiEgel e CASTELLAN JúNIOR ${ }^{21}$ para identificar o local das diferenças quando necessário.

Após a análise do efeito da dica foi também analisado se o nível de habilidade inicial influenciou a aprendizagem. Para tanto, os alunos de cada grupo foram divididos em dois subgrupos: nível baixo - quando a mediana da distância da rebatida correspondeu a 0 ; nível alto - quando a mediana da distância da rebatida foi superior a 0 . Foi utilizado o teste de Wilcoxon para a análise entre pré e pós-teste para cada nível dentro dos grupos.

Em todas as análises, o nível de significância adotado foi de 0,05 e foram realizadas utilizando-se o pacote estatístico SPSS 19.0. indicaram semelhança entre os grupos no pré-teste, mas diferenças significativas no pós-teste $\left(\chi^{2}=37,39 ; \mathrm{p}\right.$ $=0,001)$, com desempenho significativamente maior do grupo de dica perceptiva em relação aos outros. Os testes de Wilcoxon indicaram diferenças significativas entre pré e pós-teste apenas para o grupo com dica perceptiva $(Z=-4,46 ; p=0,001)$. Com base nesses resultados, pode-se inferir que os grupos partiram do mesmo estágio e apenas o grupo com dica perceptiva apresentou ganho significativo ao longo das aulas. 
TABELA 1 - Distribuição dos alunos nos estágios inicial (I), elementar (E) e maduro (M) de desenvolvimento da habilidade para os três grupos no pré e pós-teste.

\begin{tabular}{lccc}
\hline \multirow{2}{*}{ Grupo } & \multicolumn{3}{c}{ Padráo de movimento } \\
\cline { 2 - 4 } & Estágio & Pré (n\%) & Pós (n\%) \\
\hline \multirow{3}{*}{ Sem dica } & I & 92,3 & 88,5 \\
& E & 8 & 11,5 \\
Dica perceptiva & E & 0 & 0 \\
& I & 93,1 & 13,8 \\
Dica motora & M & 6,9 & 55,2 \\
& I & 82,8 & 72,4 \\
& E & 17,2 & 24,1 \\
& $\mathrm{M}$ & 0 & 3,4 \\
\hline
\end{tabular}

No que diz respeito ao tipo de rebatida, pode-se notar no pré-teste que a distribuição dos grupos diferiu (TABELA 2); enquanto que a moda do grupo sem dica foi 2 , a do grupo de dica perceptiva foi 1 e do grupo de dica motora foi 3. No entanto, no pós-teste todos os grupos apresentam a moda relativa ao estágio 3. Ao observar o desempenho entre o pré e pós-teste, nota-se que os grupos sem dica e dica perceptiva apresentaram valores modais distintos. Esses resultados foram confirmados parcialmente pelas análises inferenciais, uma vez que os testes indicaram que não houve diferença entre os grupos tanto no pré-teste quanto pós-teste, e diferença significativa entre pré e pós-teste apenas para o grupo de dica perceptiva $(Z=-2,49 ; p=0,013)$. Esses resultados permitem inferir que os grupos partiram do mesmo tipo de rebatida e apesar do grupo de dica perceptiva ter apresentado ganhos ao longo das aulas, o desempenho no pós-teste entre os grupos foi semelhante.

TABELA 2 - Moda do tipo de rebatida e sua frequência relativa de ocorrência e mediana, valores mínimos e máximos da distância da rebatida para os três grupos no pré e pós-teste.

\begin{tabular}{|c|c|c|c|c|}
\hline \multirow{2}{*}{ Grupo } & \multicolumn{2}{|c|}{ Tipo de rebatida - Estágio (n\%) } & \multicolumn{2}{|c|}{ Distância (m) - Mediana (Mín-Máx) } \\
\hline & Pré & Pós & Pré & Pós \\
\hline Sem dica & $1(69,2)$ & $3(53,8)$ & $1(0-4)$ & $0,5(0-6)$ \\
\hline Dica Perceptiva & $1(62,1)$ & $3(69,0)$ & $0(0-4)$ & $2(0-9)$ \\
\hline Dica Motora & $3(62,1)$ & $3(62,1)$ & $1(0-5,5)$ & $1(0-12)$ \\
\hline
\end{tabular}

No que diz respeito à distância da rebatida (TABELA 2), pode-se notar que no pré-teste os grupos sem dica e dica motora apresentaram valores semelhantes (mediana igual a um metro), e o grupo de dica perceptiva apresentou mediana inferior (zero) em relação aos outros grupos. No pós-teste o grupo de dica perceptiva apresentou melhores resultados em relação aos demais grupos, com uma mediana igual a dois metros. Esses resultados foram confirmados pelas análises inferenciais, uma vez que no pré-teste a estatística de Kruskal Wallis indicou diferença entre os grupos $\left(\chi^{2}=6,49 ; p=0,039\right)$, sendo que o grupo de dica perceptiva apresentou os valores significativamente mais baixos em relação aos demais grupos. O mesmo não ocorreu no pós-teste, pois não houve diferença significativa entre os grupos. Quando analisado os resultados da estatística entre pré e pós-teste, pode-se observar que houve diferença significativa apenas para o grupo de dica perceptiva $(\mathrm{Z}=-3,85 ; \mathrm{p}=$ $0,001)$. Esses resultados permitem inferir que o grupo de dica perceptiva partiu de uma posição inferior em relação aos demais, no entanto, com a realização das aulas este foi o único grupo que obteve ganhos.

\section{Análise da influência do nível inicial de habilidade}

Com base nos dados da TABELA 3, pode-se observar que no pré-teste a moda do estágio de desenvolvimento foi semelhante entre os subgrupos, independente do tipo de dica e nível inicial de habilidade. O mesmo ocorreu no pós-teste, com exceção do grupo com dica perceptiva, pois apresentou o valor modal em estágios superiores, independente do nível de habilidade inicial. Esses resultados foram confirmados pelas análises estatísticas que indicaram diferenças significativas entre pré e pós-teste apenas para os subgrupos de dicas perceptivas com nível tanto alto quanto baixo de habilidade inicial e o subgrupo de dica motora e nível de habilidade alto. Com base nesses resultados pode-se inferir que a dica perceptiva influenciou positivamente a aprendizagem independente do nível de habilidade inicial e a dica motora influenciou apenas os alunos com nível alto de habilidade motora inicial. 
TABELA 3 - Moda do estágio de desenvolvimento da habilidade e sua frequência relativa de ocorrência de acordo com o grupo de dicas e nível de habilidade inicial e os valores do teste de Wilcoxon.

\begin{tabular}{|c|c|c|c|c|c|}
\hline \multirow{2}{*}{ Grupo } & \multirow{2}{*}{ Nível de Habilidade } & \multicolumn{2}{|c|}{$\begin{array}{l}\text { Padráo de movimento } \\
\text { Estágio }(\mathbf{n} \%)\end{array}$} & \multicolumn{2}{|c|}{ Teste Wilcoxon } \\
\hline & & Pré & Pós & $\mathrm{Z}$ & $\mathbf{p}$ \\
\hline \multirow{2}{*}{ Sem dica } & Baixo & $1(100)$ & $1(100)$ & 0,1 & 0,99 \\
\hline & Alto & $1(89)$ & $1(83)$ & $-0,57$ & 0,56 \\
\hline \multirow{2}{*}{ Dica Perceptiva } & Baixo & $1(100)$ & $2(65)$ & $-3,57$ & 0,001 \\
\hline & Alto & $1(83)$ & 2 e $3(42)$ & $-2,73$ & 0,006 \\
\hline \multirow{2}{*}{ Dica Motora } & Baixo & $1(82)$ & $1(81)$ & 0,1 & 0,99 \\
\hline & Alto & $1(83)$ & $1(63)$ & $-2,0$ & 0,046 \\
\hline
\end{tabular}

Quando comparado o tipo de rebatida entre o pré e pós-teste considerando o grupo de dicas e o nível inicial de habilidade (TABELA 4), pode-se notar que os subgrupos mudaram para desempenhos superiores, com exceção de dois subgrupos com nível de habilidade inicial alto (sem dica e dica perceptiva). As análises inferenciais confirmaram esses resultados, pois indicaram que houve diferença significativa entre pré e pós-teste para todos os subgrupos de desempenho inicial baixo e apenas para o subgrupo de desempenho alto com dica motora.
Esses resultados permitem inferir que a mudança no tipo de rebatida ocorreu fundamentalmente para crianças com nível de habilidade baixo independentemente da presença ou tipo de dica, e para a dica motora em crianças com nível de habilidade alto. Considerando que o grupo sem dica com nível inicial baixo melhorou o seu desempenho no pósteste, o fato de o grupo dica motora com nível inicial baixo ter também melhorado o seu desempenho pode ser interpretado como efeito da prática em si e não do tipo de dica.

TABELA 4 - Moda do tipo de rebatida e sua frequência relativa de ocorrência de acordo com o grupo de dicas e nível de habilidade inicial e os valores do teste de Wilcoxon.

\begin{tabular}{lccccc}
\hline \multirow{2}{*}{ Grupo } & \multirow{2}{*}{ Nível de Habilidade } & \multicolumn{2}{c}{ Tipo de rebatida } & \multicolumn{2}{c}{ Teste Wilcoxon } \\
\cline { 3 - 6 } & & $\begin{array}{c}\text { Estágio (n\%) } \\
\text { Pré }\end{array}$ & $\begin{array}{c}\text { Estágio (n\%) } \\
\text { Pós }\end{array}$ & $\mathbf{Z}$ & p \\
\hline \multirow{2}{*}{ Sem dica } & Baixo & $1(100)$ & 1 e 3 (50) & $-2,0$ & 0,046 \\
\multirow{3}{*}{ Dica Perceptiva } & Alto & $2(100)$ & $3(55)$ & $-0,47$ & 0,63 \\
& Baixo & $1(100)$ & $3(65)$ & $-3,31$ & 0,001 \\
\multirow{2}{*}{ Dica Motora } & Alto & $3(92)$ & $3(75)$ & $-1,41$ & 0,157 \\
& Baixo & $1(100)$ & $3(63)$ & $-2,46$ & 0,008 \\
& Alto & $3(100)$ & $3(61)$ & $-2,64$ & 0,008 \\
\hline
\end{tabular}

Na TABELA 5 é apresentada a mediana do pré e do pós-teste de acordo com cada um dos subgrupos. Podese observar que descritivamente os únicos subgrupos que apresentaram mudança nos valores medianos ao longo das aulas foram os de dicas perceptivas com nível de habilidade tanto alto quanto baixo e o subgrupo de dicas motoras com nível de habilidade inicial baixo. Esses resultados foram confirmados pelas análises inferenciais, que indicaram esses subgrupos como os únicos com melhora da distância da rebatida entre pré e pós-teste. Com base nesses resultados, pode-se inferir que independente do nível de habilidade inicial os alunos que receberam dicas perceptivas aumentaram a distância do rebater, além dos alunos com baixo nível de habilidade que receberam dicas motoras. Mais estudos são necessários para uma melhor interpretação do resultado relativo ao efeito da dica motora para alunos que iniciam com níveis baixos. Pode ser que se tenha baixa frequência de acertos na bola, mas quando se acerta, consegue-se longas distâncias. 
TABELA 5 - Mediana da distância da rebatida de acordo com o grupo de dicas e nível de habilidade inicial e os valores do teste de Wilcoxon.

\begin{tabular}{lccccc}
\hline \multirow{2}{*}{ Grupo } & \multirow{2}{*}{ Nível de Habilidade } & \multicolumn{2}{c}{ Distância $(\mathbf{m})-$ Mediana $($ Mín-Máx) } & \multicolumn{2}{c}{ Teste Wilcoxon } \\
\cline { 3 - 6 } & & Pré & Pós & Z & p \\
\hline \multirow{2}{*}{ Sem dica } & Baixo & $0(0-0)$ & $0(0-5)$ & $-0,63$ & 0,10 \\
\multirow{3}{*}{ Dica Perceptiva } & Alto & $1(1-4)$ & $1(0-6)$ & $-0,36$ & 0,74 \\
& Baixo & $0(0-0)$ & $1,5(0-9)$ & $-3,06$ & 0,002 \\
\multirow{3}{*}{ Dica Motora } & Alto & $1(1-4)$ & $2(0-6)$ & $-2,51$ & 0,031 \\
& Baixo & $0(0-0)$ & $1(0-4)$ & $-2,37$ & 0,018 \\
& Alto & $2(1-6)$ & $1,5(0-12)$ & $-3,70$ & 0,71 \\
\hline
\end{tabular}

\section{Discussão}

No presente estudo, dicas foram manipuladas no contexto da aprendizagem do movimento na EFE. Considerando-as como conhecimentos sobre como melhorar a qualidade do movimento, buscou-se verificar seu efeito na aprendizagem da habilidade rebatida. No geral, os resultados mostraram que a rebatida foi mais bem aprendida pelos alunos que tiveram dicas perceptivas em suas aulas.

Esses resultados permitem sugerir a dica perceptiva "olho na bola" como uma informação crítica para o desempenho dos alunos na habilidade rebatida, visto que ela promoveu a aprendizagem mais bem sucedida. $\mathrm{O}$ fato de a dica perceptiva ter direcionado a atenção dos alunos para o objeto em movimento e ter possibilitado a sua interação com o ambiente, permite interpretá-la como importante conhecimento para a aprendizagem da rebatida.

Ainda, o fato de não ter havido aprendizagem em todos os grupos que receberam dicas suporta a proposição sobre a efetividade de a dica depender de ela conter informação crítica para o desempenho da tarefa. Embora dicas sejam propostas como estratégia de atenção seletiva, elas devem envolver aspectos essenciais para a realização da habilidade ${ }^{6,10-11,22-23}$.

A assunção da dica perceptiva "olho na bola" como importante conhecimento para a melhoria da qualidade do movimento ganha suporte no contexto educacional em virtude do seu potencial de transferência, ou seja, "olho na bola" poderia ser utilizado em uma gama de habilidades de rebatida em diferentes contextos como, por exemplo, no jogo de taco e no beisebol. Os resultados do presente estudo podem trazer implicações, também, para a formação profissional no sentido de permitir reflexões sobre a importância de o professor conhecer a tarefa ou o conteúdo de aprendizagem. Isto porque, caso contrário, ele poderia incorrer em erros instrucionais de não fornecer a dica mais adequada para a aprendizagem.

$O$ fato de a dica perceptiva ter influenciado positivamente a aprendizagem vem reforçar a interpretação de que as dicas de aprendizagem, para serem efetivas, devem estar atreladas à especificidade da habilidade, particularmente focando o elemento essencial da mesma $^{6,10,22}$. O rebater, por se tratar de uma habilidade aberta, requer do executante o processamento de dados perceptivos para uma tomada de decisão acertada acerca do momento correto para iniciar o movimento e assim atingir a bola no tempo e espaço adequados - timing coincidente - e não executar o movimento o mais rapidamente possível conforme sugeria a dica motora. Poderia ser pensado, ainda, que ao direcionar a atenção seletiva para o aspecto crítico da habilidade - trajetória da bola - a dica "olho na bola" poderia estimular o desenvolvimento da capacidade perceptiva que, ao longo das tentativas, possibilitaria maior atenção ao aspecto qualitativo do padrão de movimento. $O$ fato de o grupo que iniciou com nível alto de habilidade ter se beneficiado ao receber a dica motora vem reforçar essa interpretação. Isto é, para os alunos desse grupo, a dica perceptiva "olho na bola" já se fazia redundante.

Ainda, o fato de as dicas motoras terem sido efetivas para a aprendizagem dos alunos em estágios avançados relativos ao padrão de movimento reforça a sugestão sobre o direcionamento da atenção por meio da dica adequar-se às características específicas de cada aluno, da tarefa e do próprio ambiente ${ }^{6,24}$. Por exemplo, recente pesquis $\mathrm{a}^{25}$ mostrou que a dica não foi relevante em face de informações relacionadas às outras fases da habilidade de rolamento peixe 
com crianças com transtorno do desenvolvimento da coordenação.

Respeitados os limites na generalização dos resultados, as observações e interpretações que se seguem são possíveis no que se refere às implicaçôes do ensino das dicas no desenvolvimento da EFE. A dica verbal perceptiva funcionou como um conhecimento que orientou os alunos sobre como melhorar o movimento na perspectiva da aprendizagem do movimento ${ }^{1,3}$. Nesse sentido, a dica se configura como um saber escolar em potencial, cujo ensino pode contribuir para o aluno construir um conhecimento significativo para a aprendizagem do movimento. Esse conhecimento, aprendido na EFE, poderá ser utilizado em outras situações de aprendizagem da cultura de movimento para além do ambiente escolar, envolvendo esse tipo de habilidade. $\mathrm{O}$ ensino das dicas de aprendizagem pode se tornar em um dos conteúdos privilegiados da EFE, especialmente quando se considera a realidade do sistema de ensino quanto a dias e horas de aulas semanais, número de alunos por classe, espaços e materiais didáticos disponíveis ${ }^{26}$ - fatores que dificultam o alcance do outro objetivo relacionado com a aprendizagem do movimento que é melhorar a qualidade do movimento durante as próprias aulas. Para tanto, ressalta-se a necessidade de se realizar mais pesquisas com uma variedade de habilidades ensinadas na EFE, em diferentes populações escolares.

\begin{abstract}
The acquisition of striking motor skill in school Physical Education: a study of learning cues as educational content

The purpose of this study was to investigate the effects of verbal cues on the acquisition of a striking skill, focusing on their perceptual and motor aspects. The sample of this study was made of 84 public school students aged between six to eight years old from three classrooms of the same school. Each classroom constituted a group: no cue, perceptual cue and motor cue group. The experiment was carried out in three phases: pre-test, classes with specific cue and post-test. The group which received perceptual cue showed better results. Thus, it was verified that the effects of cues depend on the specificity of the task concerning its information processing demands. It was concluded that task's specificity related cue demonstrated its potential as knowledge for the students to guide them towards improvement of the quality of movement and confirms the possibility of its inclusion as a content to be taught in the school physical education.
\end{abstract}

KeY WORDS: School physical education; Motor learning; Verbal cues; Educational content.

\title{
Referências
}

1. Tani G. Desporto e escola: que diálogo ainda é possível? In: Bento JO, Constantino JM, coordenadores. Em defesa do desporto: mutações e valores em conflito. Coimbra: Almedina; 2007. p. 269-87.

2. Tani G. Perspectivas para a educação física escolar. Rev Paul Educ Fís. 1991;5:61-9.

3. Tani G. Abordagem desenvolvimentista: 20 anos depois. Rev Educ Fís. 2008;19:313-33.

4. Halverson LE. The young child... The significance of motor development. In: Engstrom G, editor. The significance of the young child's motor development. Washington: National Association for the Education of Young Children; 1971.

5. Ericsson KA, Krampe RT, Tesch-Römer C. The role of deliberate practice in the acquisition of expert performance. Psychol Rev. 1993;100:363-406.

6. Landin DK. The role of verbal cues in skill learning. Quest. 1994;46:299-313.

7. Ladewig I. A importância da atenção na aprendizagem de habilidades motoras. Rev Paul Educ Fís. 2000;14:62-71.

8. Magill RA. Motor learning and control: concepts and applications. 9th ed. Boston: Mcgraw-Hill; 2010.

9. Schmidt RA, Lee T. Motor control and learning: a behavioral emphasis. 5th ed. Champaign: Human Kinetics; 2011.

10. Fronske H. Teaching cues for sport skills for secondary school students. 5th ed. Boston: Benjamin Cummings; 2012. 
11. Marques MTSP. Dicas verbais na aprendizagem de uma habilidade motora: foco no comportamento e na interação entre os componentes [dissertação]. São Paulo (SP): Universidade de São Paulo, Escola de Educação Física e Esporte; 2012.

12. Freudenheim AM, Iwamizu JS, Santos S. Da pesquisa sobre instrução à intervenção profissional. In: Corrêa UC. Pesquisa em comportamento motor: a intervenção profissional em perspectiva. São Paulo: EFP/EEFEUSP; 2008. p. 231-9.

13. Masser LS. Critical cues help first-grade students' achievement in handstands and forward rolls. J Teach Phys Educ. 1993;12:301-12.

14. Buchanan AM, Briggs J. Making cues meaningful: a guide for creating your own. Teach Elem Phys Educ. 1998;8:16-8.

15. Roach NK, Burwitz L. Observational learning in motor skill acquisition: the effect of verbal directing cues. In: Watkins J, Riley T, Burwitz L, editors. Sport science: proceedings of the VIII Commonwealth and International Conference on Sport, Physical Education, Dance, Recreation, and Health; 1986. New York: Spon; 1986. p. 349-54.

16. Oliveira JA. Aquisição de uma habilidade motora básica: a prática sistemática em foco [tese]. São Paulo (SP): Universidade de São Paulo, Escola de Educação Física e Esporte; 2006.

17. Williams HG. Perceptual and motor development. New Jersey: Prentice Hall; 1983.

18. Tani G, Manoel EJ, Kokubun E, Proença JE. Educação física escolar: fundamentos de uma abordagem desenvolvimentista. São Paulo: EPU/EDUSP; 1988.

19. Gallahue DL, Ozmun JC, Goodway JD. Understanding motor development: infants, children, adolescents, adults. 7th ed. Boston: McGraw-Hill Education; 2011.

20. Silveira SR. Aquisição de habilidades motoras na educação física escolar: um estudo das dicas de aprendizagem como conteúdo de ensino [tese]. São Paulo (SP): Universidade de São Paulo, Escola de Educação Física e Esporte; 2010.

21. Siegel S, Castellan Júnior J. Estatística não-paramétrica para ciências do comportamento. São Paulo: Artmed; 2006.

22. Pasetto SC, Araújo P, Corrêa UC. Efeitos de dicas visuais na aprendizagem do nado crawl para alunos surdos. Rev Port Ciênc Desporto. 2006;6:14-9.

23. Medina-Papst J, Ladewig I, Marques I. Dicas de aprendizagem na aquisição de habilidades motoras: uma revisão. Rev Educ Fís. 2009;20:625-35.

24. Bôscolo EFM, Santos LM, Oliveira SL. Natação para adultos: a adaptação ao meio aquático fundamentada no aprendizado das habilidades motoras aquáticas básicas. Rev Educ. 2011;6:21-8.

25. Medina-Papst J, Ladewig I, Rodacki AF, Marques I. Dicas de aprendizagem auxiliam as crianças com TDC na aquisição de uma habilidade motora complexa? Rev Bras Ciênc Esporte. 2012;34:477-94.

26. Tokuyoshi JH, Bigotti S, Antunes FHC, et al. Retrato dos professores de educação física das escolas estaduais do Estado de São Paulo. Motriz: Rev Educ Fís. 2008;14:418-28.

\begin{tabular}{r|r} 
ENDEREÇO & \\
Sérgio Roberto Silveira & \\
R. Mário Maglio, 198 & Recebido para publicação: 08/05/2012 \\
05530-050 - São Paulo - SP - BRASIL & Aceito: 19/10/2012 \\
e-mail: ssilveira@usp.br & \\
&
\end{tabular}

